\title{
Bone mineral density, collagen type $1 \alpha 1$ genotypes and bone turnover in premenopausal women with diabetes mellitus
}

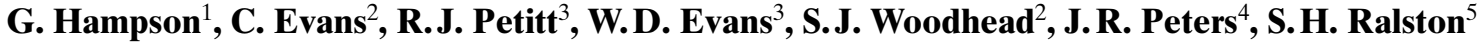 \\ ${ }^{1}$ Department of Chemical Pathology, St Thomas' Hospital, London, UK \\ ${ }^{2}$ Department of Medical Biochemistry, University Hospital of Wales, Cardiff, UK \\ ${ }^{3}$ Department of Medical Physics, University Hospital of Wales, Cardiff, UK \\ ${ }^{4}$ Department of Medicine, University Hospital of Wales, Cardiff, UK \\ ${ }^{5}$ Department of Medicine and Therapeutics, Polwarth Building, Aberdeen, UK
}

Summary Osteopenia is a recognised complication of diabetes mellitus which could be due to abnormal bone turnover or disturbances in the calcium/parathyroid hormone/vitamin D axis or both. Genetic factors also play an important part in determining bone mass although this has not been studied in diabetes. Recently a polymorphism of the collagen type $1 \alpha 1$ (COL1A1) gene has been shown to be associated with low bone mass in British women. To identify subjects with diabetes who may be at risk of developing osteoporosis and fractures, we analysed bone mineral density in relation to the biochemical markers of bone turnover, calcium homeostasis and the COL1A1 genotype in a group of premenopausal women with Type I (insulin-dependent) diabetes mellitus $(n=31)$, Type II (non-insulin dependent) diabetes mellitus $(n=21)$ and control subjects $(n=20)$. Bone mineral density was lower at the femoral neck in the subjects with Type I diabetes $(p=0.08)$ as were serum 25-hydroxyvitamin D compared with control subjects $(p=0.023)$ and this was negatively correlated with serum collagen type $1 \mathrm{C}$-terminal propeptide $(r=-0.56, p<0.001)$. Bone mineral den- sity in Type II diabetes was not different from control subjects, after correction for body mass index. Bone resorption was, however, raised in the Type II diabetic subjects as reflected by the higher urinary deoxypyridinoline values $(p=0.016)$ and lower collagen type $1 \mathrm{C}$-terminal propeptide:deoxypyridinoline ratio $(p=0.04)$. In the whole group studied, subjects with the COL1A1 's' genotype had lower bone mineral density at the femoral neck $(p=0.01)$ which was partly attributable to a lower body mass index. Following multiple regression analysis body mass index and collagen type $1 \mathrm{C}$-terminal propeptide concentrations remained determinants of bone mass at all three sites, whereas genotype appeared to be a predictor of bone mass at the femoral neck only. We conclude that measurement of these variables could prove useful in firstly identifying those diabetic women at risk of osteoporosis and secondly guiding therapeutic intervention. [Diabetologia (1998) 41: 1314-1320]

Keywords Diabetes mellitus, bone, COL1A1 polymorphism, vitamin $\mathrm{D}$, parathyroid hormone, bone markers.
Diabetic osteopenia is a recognised but neglected complication of diabetes mellitus. Reduced bone

Received: 2nd March 1998 and in revised form: 27 April 1998

Corresponding author: Dr. G. Hampson, Department of Chemical Pathology, St Thomas' Hospital, Lambeth Palace Road, London SE1 7EH, UK

Abbreviations: $25-(\mathrm{OH}) \mathrm{D}, 25-\mathrm{Hydroxyvitamin} \mathrm{D;} \mathrm{CICP,} \mathrm{col-}$ lagen type $1 \mathrm{C}$-terminal propeptide; COL1A1, collagen type 1 $\alpha 1 ; \mathrm{CV}$, coefficient of variation; PCR, polymerase chain reaction. mass and an overall twofold increase in fracture rate has been shown to occur in Type I (insulin-dependent) diabetes mellitus [1]. The situation in Type II (non-insulin-dependent) diabetes mellitus is less clear-cut, with reports of increased, decreased or unaltered bone mass reflecting the underlying heterogeneity of Type II diabetes [2]. The pathogenesis of diabetic osteopenia remains unclear. The reduction in bone mass could be due, in part, to a failure to reach peak bone mass especially in younger subjects with Type I diabetes [3] and partly to abnormal bone turnover [4]. In adults net bone loss has been found with a 
rate of bone loss three times higher in patients in whom endogenous insulin concentrations had decreased [5]. Insulin is regarded as a systemic regulator of bone formation [6].

In addition to the direct effect of insulin deficiency, other metabolic disturbances such as abnormalities of the vitamin $\mathrm{D} /$ parathyroid hormone axis can also contribute to the diabetic osteopathy [7]. Several studies in experimental rat models $[8,9]$ and in humans with diabetes [10] have suggested that an impairment of the vitamin $\mathrm{D} /$ parathyroid hormone endocrine system could have a role in bone loss.

As the determinants of bone mass are predominantly genetic, it is also likely that genetic factors will influence the development of osteopenia in diabetic patients. Genetic factors have been shown to influence the development of postmenopausal osteoporosis [11] and the vitamin D receptor (VDR) gene has been implicated as one candidate for the regulation of bone mass [12]. The relation between the vitamin D receptor gene and bone mineral density is controversial [13]. Of the many genes that could potentially regulate bone mineral density, type 1 collagen has been the first to be suspected of putatively underlying the genetic effect on bone mass as mutations in its coding region give rise to severe osteoporosis and osteogenesis imperfecta. Recent studies have shown that a novel polymorphism in the collagen type $1 \alpha 1$ (COL1A1) gene is overrepresented in patients with osteoporotic fractures and is associated with reduced bone density [14]. This has not been studied in patients with diabetes.

As improvements in the care of diabetic patients prolong their life expectancy, the importance of increased fracture risk is likely to become greater. Diabetes per se could add to the postmenopausal and age-related bone loss although prospective studies are lacking. To devise effective clinical and therapeutic strategies it is important to identify those diabetic women at increased risk of developing osteoporosis. The aim of this study was firstly to assess bone mineral density, secondly the biochemical markers of bone turnover, thirdly COL1A1 gene polymorphism and fourthly calcium homeostasis in a group of premenopausal women with diabetes to identify those indices which would be the best predictors of osteoporosis and fracture risk in diabetic women. This cross-sectional study should also provide preliminary information on whether any of these variables could guide clinical management in this group and be included in a 'risk factor profile' in future prospective studies of bone metabolism in diabetes.

\section{Subjects and methods}

Subjects. We studied 72 premenopausal women who were divided into 3 groups: group 1 was the Type I diabetic group and comprised 31 women, group 2 was the Type II diabetic group and comprised 21 women, group 3 was the control group and comprised 20 nondiabetic women matched for age. Exclusion criteria were heavy smoking ( $>10$ cigarettes/ day), an alcohol intake of greater than 12 units/week, renal impairment (serum creatinine $>110 \mu \mathrm{mol} / \mathrm{l}$ ), abnormal urinary albumin excretion (urine albumin $>30 \mathrm{mg} / 24 \mathrm{~h}$ ), any drug treatment which can influence bone metabolism such as thyroid hormones, steroids, products containing vitamin $\mathrm{D}$ or its derivatives, calcium and magnesium. All subjects were Caucasians and physically active. There was no difference in the level of physical activity between the groups. The diabetic women who met all eligibility criteria were selected from the diabetic clinic database of patients and invited to participate in the study. The study was performed in accordance with the Helsinki declaration and approved by the local ethics committee. The subjects were seen in the investigation unit on the metabolic ward and informed consent was obtained.

Clinical data on all subjects are summarised in Table 1. The subjects with Type I diabetes were receiving twice daily injections of a short-acting and an intermediate-acting insulin. The prevalence of diabetic complications in this group was low. None of the patients had diabetic nephropathy or clinical evidence of neuropathy. Only three of the patients in Group 1 had proliferative retinopathy and they had received successful retinal photocoagulation treatment. The rest of the patients in this group had either no or background retinopathy. The patients with Type II diabetes were receiving oral hypoglycaemic agents (metformin or sulphonylureas or both). Two patients in this group were also being treated with lipid lowering drugs. None of the patients with Type II diabetes had any evidence of nephropathy or neuropathy. Only one patient in Group 2 had proliferative retinopathy.

Biochemical assays. Random blood samples were taken and a 24-h urine collection obtained. Serum and urine concentrations of urea, creatinine, electrolytes, calcium, phosphate and magnesium were measured by standard laboratory techniques on the Hitachi 747 (Boehringer Mannheim, Mannheim, Germany). We measured $\mathrm{HbA}_{1 \mathrm{c}}$ by high performance liquid chromatography using the Bio-Rad variant assay system (Bio Rad Labs. Hemel Hempstead Herts U. K.). Serum parathyroid hormone was measured by an immunochemiluminometric assay (Ciba Corning Diagnostics Ltd. Halstead, Essex, U. K.). Interassay coefficient of variation (CV) was $12.4 \%$. Serum 25 -hydroxyvitamin D (25- $(\mathrm{OH}) \mathrm{D})$ was determined by radioimmunoassay (Diasorin Ltd Wokingham Berkshire U.K., Minn., USA). Inter-assay CV was 15 and $11 \%$ at serum $25-(\mathrm{OH}) \mathrm{D}$ concentrations of 13.8 and $53.7 \mathrm{ng} / \mathrm{ml}$, respectively. Serum and urine samples for the determination of the biochemical bone markers were frozen at $-20^{\circ} \mathrm{C}$ until analysis. Blood samples was also kept at $-20^{\circ} \mathrm{C}$ for DNA extraction.

Bone turnover was assessed by measuring serum collagen type $1 \mathrm{C}$-terminal propeptide (CICP) and urinary deoxypyridinoline. The procollagen molecule (CICP) reflects collagen type 1 formation and subsequently bone formation. Deoxypyridinoline is a bone specific collagen breakdown product released during resorption of bone tissue and is therefore useful as a marker of bone resorption. Serum CICP was determined in duplicate by an immunometric assay using the Prolagen $\mathrm{C}$ kit (Metra Biosystems Ltd, Great Haseley Oxon U. K.). Interassay CV was 1.3 and $10 \%$ at CICP concentrations of 4.5 and $30.5 \mathrm{ng} / \mathrm{ml}$, respectively. Deoxypyridinoline was measured in duplicate in the 24-h urine collections by the Pyrilinks-D assay (Metra Biosystems Ltd). We analysed 24-h urine collections as opposed to random samples to minimise variation in excretion. Inter-assay CV was 14 and $4.5 \%$ at deoxypyridinoline concen- 
Table 1. Summary of data on all subjects

\begin{tabular}{llll}
\hline & Type I & Type II & Subjects \\
\hline Subjects $(n)$ & 31 & 21 & 20 \\
Age $($ years $)$ & $42.4(8.9)$ & $42.5(5.5)$ & $41.8(5.2)$ \\
Duration of diabetes (years) & $20.2(10.5)$ & $7.6(5)$ & - \\
$\mathrm{HbA}_{1 \mathrm{c}}(\%)$ & $9.2(1.4)$ & $8.5(1.9)$ & $5.3(0.5)$ \\
$\mathrm{BMI}\left(\mathrm{kg} / \mathrm{m}^{2}\right)$ & $25.6(3.2)$ & $34.4(5.7)^{1}$ & $25(5.5)$ \\
BMD lumbar spine $\left(\mathrm{g} / \mathrm{cm}^{2}\right)$ & $1.064(0.16)$ & $1.21(0.11)$ & $1.108(0.15)$ \\
BMD neck of femur $\left(\mathrm{g} / \mathrm{cm}^{2}\right)$ & $0.8(0.15)^{5}$ & $0.936(0.14)$ & $0.891(0.12)$ \\
BMD forearm $\left(\mathrm{g} / \mathrm{cm}^{2}\right)$ & $0.58(0.05)$ & $0.623(0.05)^{4}$ & $0.586(0.035)$ \\
Serum CICP $\left(\mathrm{ng} / \mathrm{ml}^{2}\right)$ & $102.6(41.6)$ & $78.2(22.7)$ & $88.9(28.2)$ \\
Dpd/creatinine $(\mathrm{nmol} / \mathrm{mmol})$ mean $($ range $)$ & $4.83(2-32.4)$ & $16.4(6.10)^{2}$ & $4.08(2.7-8.4)$ \\
CICP/Dpd ratio mean $(\mathrm{range})$ & $27.2(2.6-50.7)$ & $2.3(0.1)$ & $23.3(8.6-40.8)$ \\
Serum calcium $(\mathrm{mmol} / \mathrm{l})$ & $2.23(0.06)$ & $1.08(0.2)$ & $2.31(0.1)$ \\
Serum phosphate $(\mathrm{mmol} / \mathrm{l})$ & $1.01(0.2)$ & $2.96(1.9)$ & $1.03(0.16)$ \\
Serum PTH $(\mathrm{pmol} / \mathrm{l})$ & $2.6(1.5)$ & $0.75(0.06)^{1}$ & $3.0(1.0)$ \\
Serum magnesium $(\mathrm{mmol} / \mathrm{l})$ & $0.79(0.05)$ & $21(10)$ & $0.82(0.057)$ \\
Serum $25-(\mathrm{OH}) \mathrm{D}(\mathrm{ng} / \mathrm{ml})$ & $16.9(6.3)^{3}$ & $24.5(13)$
\end{tabular}

Results are expressed as mean (SD) except for dpd and CICP/ dpd ratio.

Differences between groups were analysed by analysis of variance followed by Scheffe correction and by the Mann-Whitney $\mathrm{U}$ test for $\mathrm{Dpd} /$ creatinine and $\mathrm{CICP} / \mathrm{Dpd}$. BMD was un- corrected for BMI. ${ }^{1} p=0.000 ;{ }^{2} p=0.016 ;{ }^{3} p=0.023 ;{ }^{4} p=$ $0.04 ;{ }^{5} p=0.08$ compared with control subjects.

BMD, bone mineral density; Dpd, deoxypyridinoline; PTH, parathyroid hormone trations of 16 and $102 \mathrm{nmol} / \mathrm{l}$, respectively. Results were expressed as creatinine ratios.

$D N A$ analysis. Isolated DNA from whole blood using the DNA extraction kit from Quiagen Ltd (Dorking, Surrey. U.K.) and amplified DNA sequences by polymerase chain reaction (PCR). To amplify the COL1A1 gene polymorphism, PCR was carried out using the protocol described previously [14]. After PCR the reaction products were digested overnight at $37^{\circ} \mathrm{C}$ with $\mathrm{MScI}$ and analysed by agarose gel electrophoresis. Genotypes were determined according to the digestion pattern of the PCR products. Alleles were coded 'S' or ' $\mathrm{s}$ ' (absence or presence of restriction site).

Bone densitometry. Bone mineral density at the spine (L1-L4), left hip (neck of femur) and the left forearm (total radius) was measured by dual-energy X-ray absorptiometry (DXA) using the Hologic QDR 1000/W DXA scanner Waltham MA. USA. The measurement $\mathrm{CV}$ was $1 \%$ at the forearm and spine and $1.8 \%$ at the femoral neck. The World Health Organisation criteria for the diagnosis of osteoporosis and osteopenia were applied. Osteoporosis was defined as a bone mineral density of less than $-2.5 \mathrm{SD}$ below the young normal mean (T score). Subjects with osteopenia had a ' $T$ ' score between -1 and -2.5 .

Statistical analysis. A one-way analysis of variance followed by Scheffe test correction was used to compare parametric data between the subjects with Type I diabetes, Type II diabetes and control subjects. Mann-Whitney U test was used for nonparametric data. Linear regression analysis (univariate and stepwise multiple linear regression) was made to assess the dependence of bone mineral density on the independent variables such as clinical characteristics, biochemical markers of bone turnover and calcium homeostasis and genotype. Pearson's Chi-squared test was used to assess the frequency distribution of the different genotypes. Statistical analyses were made using the statistical software STATA 5, STATA Corp (1997) College Station Tex., USA.

\section{Results}

Bone mineral density. The three groups of subjects were matched for age. Body mass index (BMI) was significantly higher in the group with Type II diabetes compared with control subjects. There was no significant difference in BMI between the Type I diabetic group and control subjects. The results are summarised in Table 1. Bone mineral density, uncorrected for BMI, was significantly higher at the forearm only in the subjects with Type II diabetes compared with control subjects. After correction for BMI there was no difference in bone mineral density at any site between subjects with Type II diabetes and control subjects. Bone mineral density at the neck of femur in the Type I diabetic group tended to be lower than in control subjects' mean (SD) 0.8 $\mathrm{g} / \mathrm{cm}^{2}(0.12)$ compared with $0.891 \mathrm{~g} / \mathrm{cm}^{2}(0.15)$ although the results just failed to reach significance $(p=0.08)$. Four of the Type I diabetic $(13 \%)$ patients had $\mathrm{T}$ scores of less than -2.5 at the hip (Germany) (Figure 1). After univariate analysis, there was a positive association of bone mineral density with BMI and Type II diabetes at all three sites. After correction for BMI, however, the association between bone mineral density and Type II diabetes no longer remained significant. A significant negative association was found between bone mineral density at the neck of femur and Type I diabetes which was unaffected by correction for BMI. There was no significant association between $\mathrm{HbA}_{1 \mathrm{c}}$, age, duration of diabetes, serum calcium and magnesium, parathyroid hormone and bone mineral density at any site in the diabetic patients. Significant associations are shown in Table 2. After stepwise multiple 


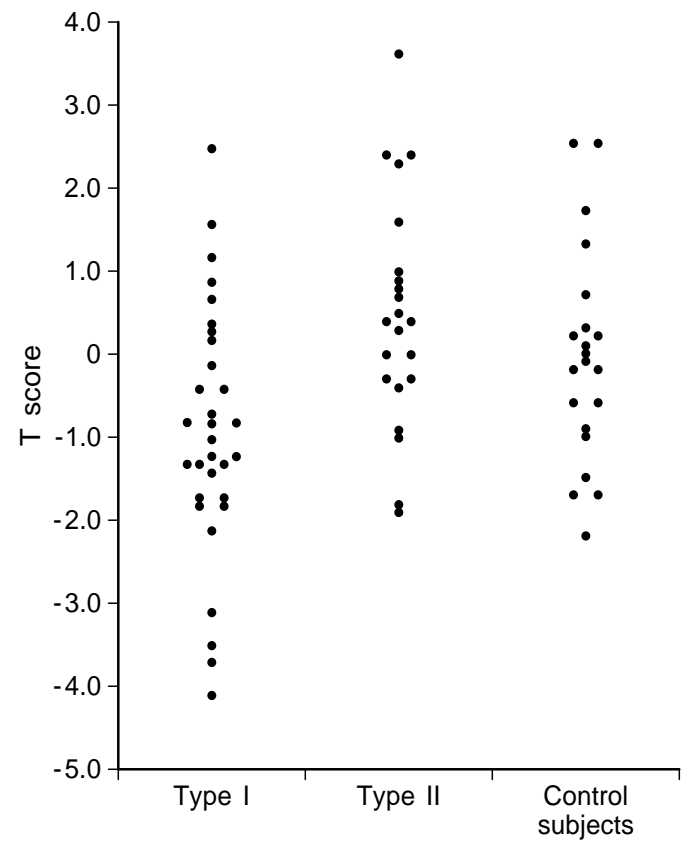

Fig. 1. ' $T$ ' score at the femoral neck in the subjects with Type I diabetes, Type II diabetes and control subjects

linear regression as summarised in Table 2, BMI remained a strong predictor of bone mineral density at the spine and neck of femur. In the whole group $24 \%$ of the variability in bone mineral density at the neck of femur and lumbar spine was explained by $\operatorname{BMI}(p=0.045)$.
Bone markers. Serum CICP was not significantly different in the Type I diabetes and Type II diabetic groups compared with control subjects (Table 1). There was a significant negative correlation between serum 25-(OH) D and CICP in the subjects with Type I diabetes (Fig.2). Deoxypyridinoline was not significantly different in the Type I diabetic group compared with control subjects. It was, however, significantly higher in the Type II diabetic group than in the control group and CICP:deoxypyridinoline ratio was significantly lower in the Type II diabetic group than in the control subjects. Bone mineral density was negatively associated with CICP concentrations at all three sites. This association remained significant at the neck of femur and lumbar spine after stepwise multiple linear regression (Table 2).

COL1A1 genotypes. There was no difference in allele frequency between the Type I diabetic, Type II diabetic and control groups as is summarised in Table 3. In the whole group studied, $65.3 \%(n=47)$ of the women had the 'SS' genotype and $34.7 \% \quad(n=25)$ the 'Ss' or 'ss' genotypes. There was differential representation of COL1A1 alleles in patients with osteopenia at the neck of femur only. The 'Ss' and 'ss' genotypes were significantly over-represented in osteopenic patients (Fig. 3). Of the subjects who were osteopenic at the neck of femur, 12 out of 22 $(54.5 \%)$ had the 's' allele. Only 13 out of the 50 subjects $(26 \%)$ with a $\mathrm{T}$ score of greater than -1 had the 's' allele. A similar trend was also observed in each

Table 2. Summary of significant associations with bone mineral density after univariate and stepwise multiple regression analysis

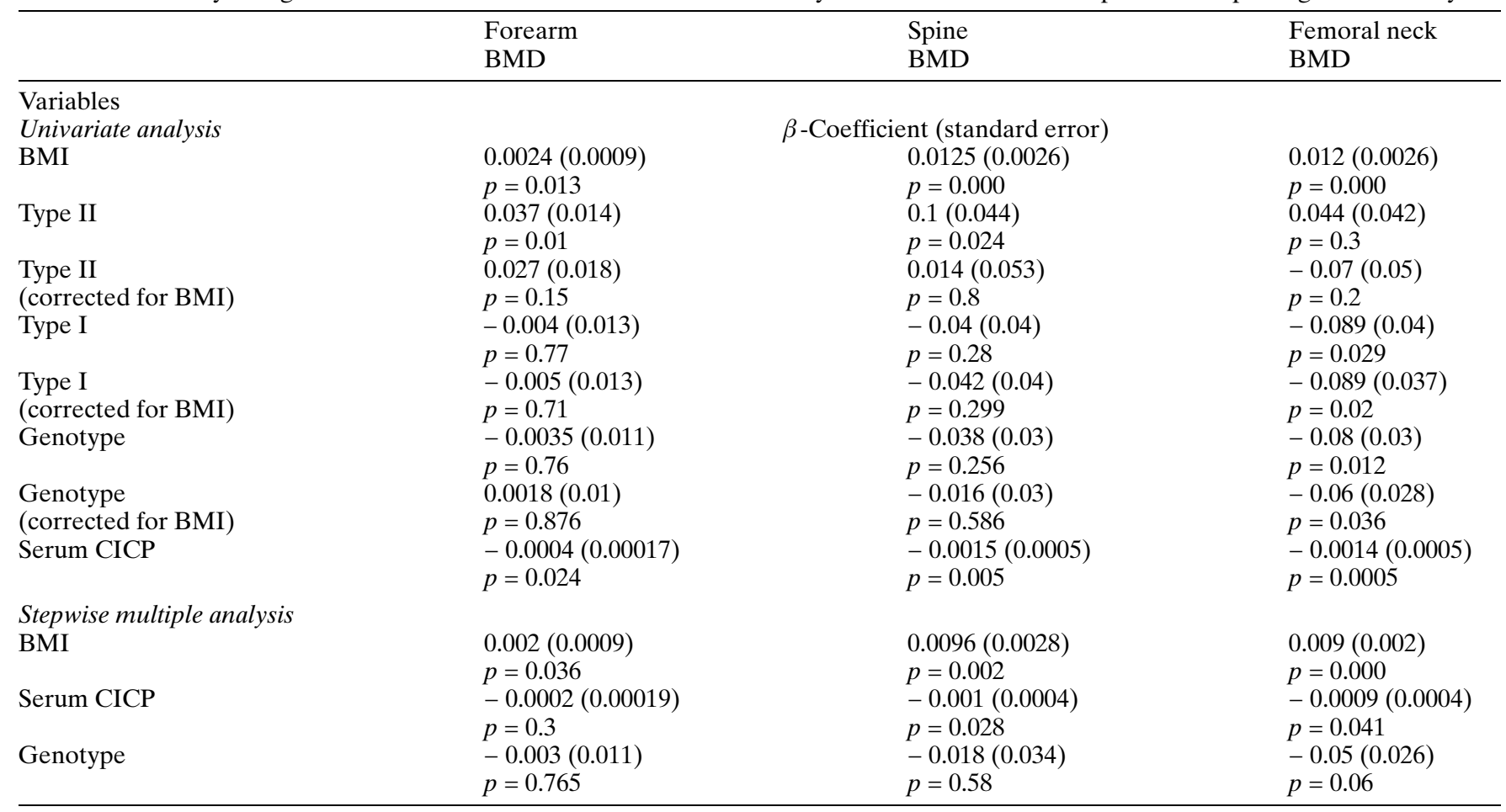

BMD, bone mineral density 


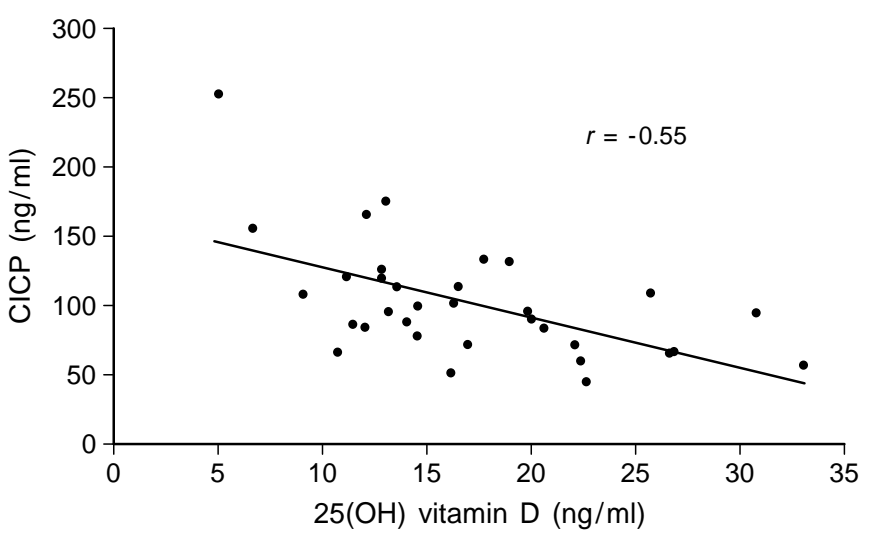

Fig. 2. The correlation between serum CICP and 25(OH)vitamin $\mathrm{D}$ concentrations in women with Type I diabetes $(r=-0.56, p<0.001)$

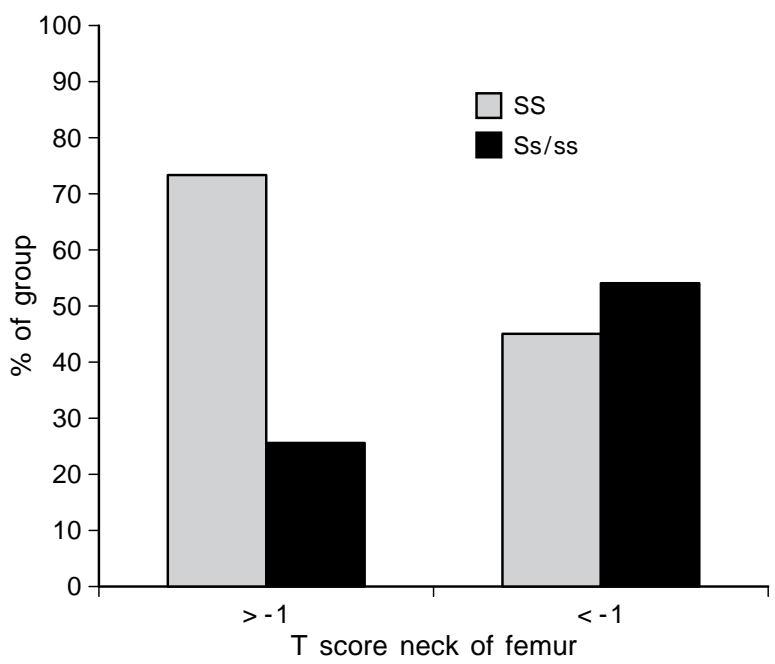

Fig.3. Overrepresentation of COL1A1 's' allele in subjects with T score $<-1$ at the neck of femur $(n=22)$ compared with patients with $\mathrm{T}$ score $>-1 \quad(n=50)$. Number of subjects in each group were SS:10 Ss/ss:12 (T score $<-1$ ) compared with $\mathrm{SS}: 37 \mathrm{Ss} / \mathrm{ss}: 13(\mathrm{~T}$ score $>-1)\left(\chi^{2}\right.$ test $\left.=4.8, p=0.029\right)$

group with the 's' genotype present in over $50 \%$ of patients with $\mathrm{T}$ score less than -1 as compared with about $25 \%$ in patients with $\mathrm{T}$ score more than -1 . Three out of the four patients with Type I diabetes with $\mathrm{T}$ score less than -2.5 at the femoral neck had the 's' allele. Bone mineral density at the neck of femur was $10 \%$ lower in the 'Ss' or 'ss' subjects when compared with those with the 'SS' genotypes (mean bone mineral density of $0.9 \mathrm{~g} / \mathrm{cm}^{2}$ vs $0.81 \mathrm{~g} / \mathrm{cm}^{2}$, $p=0.01)$. There was also a significant difference in BMI between the genotypes. It was significantly lower in 'Ss' and 'ss' compared with 'SS' subjects $(p=0.05)$. Linear regression analysis showed a significant association between bone mineral density at the neck of femur and genotype which remained significant after correction for BMI. After stepwise multiple linear regression the association of bone mineral
Table 3. Genotype distribution in the whole group and by diagnosis and summary of bone mineral density, BMI data and age by genotype

\begin{tabular}{lll}
\hline & \multicolumn{2}{l}{ Genotypes } \\
\cline { 2 - 3 } & SS & Ss/ss \\
\hline $\begin{array}{l}\text { Number of patients } \\
n(\%)\end{array}$ & $47(65.3 \%)$ & $25(34.7 \%)$ \\
$\begin{array}{l}\text { Distribution by disease } \\
\text { Type I (\%) }\end{array}$ & \\
Type II (\%) & $64.5 \%$ & $35.5 \%$ \\
Control subjects $(\%)$ & $66.6 \%$ & $33.3 \%$ \\
BMD (g/cm $\left.{ }^{2}\right)$ & $65 \%$ & $35 \%$ \\
mean (SD) & & \\
Forearm & & \\
Lumbar Spine & $0.59(0.047)$ & $0.59(0.057)$ \\
Neck of femur & $1.13(0.15)$ & $1.09(0.17)$ \\
BMI (kg/m $\left.{ }^{2}\right)$ & $0.9(0.15)$ & $0.81(0.15)^{1}$ \\
Age (years) & $29(6.4)$ & $25.7(5.3)^{2}$ \\
\hline
\end{tabular}

${ }^{1} p=0.01,{ }^{2} p=0.05$ compared with SS genotype.

$\mathrm{BMD}$, bone mineral density

density at the femoral neck with genotype still approached significance $(p=0.06)$. There was no significant genotype-specific differences in CICP or deoxypyridinoline.

In predicting bone mass in this group, multiple regression analysis showed that $40 \%$ of the variability in bone mineral density at the femoral neck can be explained by BMI, Type I diabetes, serum CICP and genotype. At the lumbar spine, $36 \%$ of the variability could be explained by BMI and serum CICP concentrations.

Vitamin D/parathyroid hormone axis. No significant difference was observed in serum calcium, phosphate and parathyroid hormone between the three groups (Table 1). Serum magnesium was significantly lower in the Type II diabetic group compared with control subjects. Urinary calcium, phosphate and magnesium did not differ significantly between the three groups (data not shown). The Type I diabetic group had a significantly lower serum concentration of $25-(\mathrm{OH}) \mathrm{D}$ compared with control subjects. Two of the four Type I diabetic patients with a $\mathrm{T}$ score of less than -2.5 at the neck of femur had very low serum 25 $(\mathrm{OH})$ D concentrations $(<5.0$ and $6.6 \mathrm{ng} / \mathrm{ml})$.

\section{Discussion}

Osteoporosis causes considerable mortality and morbidity leading to an enormous financial burden on the health services. Whilst population based screening cannot be justified at present, identification and close follow-up of "high-risk" groups provides a logical approach to the prevention and treatment of the 
condition. Women with diabetes may be considered a subgroup at "high-risk" of developing osteoporosis. A reduction in bone mass and a twofold increase in fracture risk have been shown to be associated with Type I diabetes [1]. In the metabolically more heterogeneous group of Type II diabetes, conflicting results regarding bone mass have been observed. Differences in body mass index, variation in the degree of insulinopenia or beta-cell failure or both in this group could explain the inconsistent findings [15]. The establishment of a means of screening this group is important considering the increased life expectancy of diabetic patients. Diabetic women are likely to be more at risk of fracture since diabetes per se would add to the additional post-menopausal and age-related bone loss, although prospective data are not available to date. This study assessed bone mineral density, bone turnover, the COL1A1 gene polymorphism and the calcium/parathyroid hormone/vitamin D axis in patients with Type I and Type II diabetes. The aim was to identify the most useful determinants in the assessment of bone metabolism and fracture risk in diabetic women. These variables can be used to guide firstly therapeutic intervention and secondly the design of future prospective studies in this group. A premenopausal group was studied to minimise the confounding effect of the menopause and to obtain baseline data. We observed lower bone mineral density at the femoral neck and serum concentrations of $25(\mathrm{OH})$ vitamin $\mathrm{D}$ in women with Type I diabetes. Both factors are associated with an increased risk of fractures. Bone resorption was significantly raised in Type II diabetes. This study also showed an association for the first time between COL1A1 polymorphism and bone mineral density at the femoral neck in patients with diabetes. Body mass index and serum CICP were significantly associated with bone mass at the lumbar spine and neck of femur.

This study confirmed the association of low bone mineral density at the femoral neck and Type I diabetes [16]. In contrast to other studies [17] duration of disease did not seem to be an important factor in the development of osteopenia at the hip. This would imply that the acute and major bone loss occurs early in the course of the disease [18]. This is likely to be a factor in the Type I diabetic women as most showed this loss in childhood. This pattern of bone loss or of failure to achieve peak bone mass may be due to the absolute or near absolute deficiency of insulin which accompanies the onset of diabetes mellitus which is then corrected with the introduction of insulin treatment in this group. Nevertheless, the initial reduction in bone mass would put them at higher risk of fracture. It is noteworthy that a reduction in bone mineral density is seen only at the femoral neck in Type I diabetes. The fact that the femoral neck is a compartment comprising of both trabecular and cortical bone, whereas the lumbar spine consists mainly of trabecular bone, might explain the discrepancy in bone mineral density changes, at least, at these two sites.

In the patients with Type II diabetes the higher bone mineral density at the forearm can be attributed to the higher body mass of this group. Body mass index was found to be an important determinant of bone mineral density at all sites as reported previously [19]. Bone turnover was, however, significantly different compared with control subjects and those with Type I diabetes. It is the generally held view that diabetic osteopenia is a low turnover condition [20,21]. Our results in the patients with Type II diabetes suggest, however, increased bone resorption. In contrast to previous studies, the increased bone resorption was not related to medium-term metabolic control [22]. It has been suggested that bone loss is less in patients with Type II diabetes who are receiving insulin than those receiving oral hypoglycaemic agents [23]. This may partly explain our findings as the patients with Type II diabetes in our study were receiving oral hypoglycaemic agents. This is compatible with the view that insulin is required for the maintenance of bone integrity. The pathogenetic mechanisms of increased bone resorption in this group are likely to be multifactorial, given the heterogeneity of the condition and merit further investigations. An inverse association between serum CICP and bone mineral density was observed. This is surprising as CICP is generally considered to be a marker of bone formation. Our results would, however, indicate that CICP like osteocalcin is a marker of bone turnover. Measurement of the biochemical markers of bone metabolism could thus be useful in this group to identify those women with Type II diabetes who have an imbalance of bone remodelling and are at risk of bone loss. Although a reduction in bone mineral density is not apparent at this stage in the premenopausal women with Type II diabetes, should this increased bone turnover persist in the very long-term, an appreciable negative effect on bone mass might be expected after the menopause.

The major determinant of bone mass is genetic [11] with estimates of heritability ranging from 50 to $70 \%$. Genetic factors have been shown to influence the development of postmenopausal osteoporosis. Recent studies have shown that the novel polymorphism in the COL1A1 gene is overrepresented in patients with an osteoporotic fracture and is associated with reduced bone density. This polymorphism can also influence the development of osteopenia in diabetic women, although there are no studies to date. COL1A1 genotype was associated with bone mineral density at the hip and therefore appears to be predictive of bone mass at this site. Our results are in contrast to a previous study [14] where an association between COL1A1 polymorphism and bone mineral density at the spine only was observed in a group of mostly postmenopausal women. Firstly the difference in the results between the 2 studies could be ex- 
plained partly by the difference in the menopausal status or age or both of the subjects studied. It could be that COL1A1 polymorphism is associated with peak bone mass at the femoral neck. Secondly the association between bone mineral density and the COL1A1 genotypes could have become more apparent in our study because of the added influence of diabetes mellitus (Type I) in determining bone mineral density at the femoral neck. The results also suggest that the effect of the COL1A1 genotype on bone mineral density can be partly mediated by its genetic effect on body size. Analysis of the COL1A1 polymorphism in this group could thus help in identifying those diabetic women at risk of osteoporosis.

Diabetic osteopenia has also been related to dysregulation of the calcium/parathyroid hormone/vitamin D axis [9, 10, 24]. Risk factors could also include low or suboptimal calcium/vitamin D intake which patients with diabetes might be at risk of as a result of the low fat 'diabetic diet'. The lower serum 25$(\mathrm{OH}) \mathrm{D}$ concentrations in the subjects with Type I diabetes are unlikely to be due to seasonal variation as they were recruited at random at the same time of the year as the control subjects. Low vitamin D concentrations are associated with high bone turnover. This could potentially be detrimental especially in those patients who are already osteopenic. The lower serum magnesium in the patients with Type II diabetes can also lead to abnormalities in bone metabolism. Hypomagnaesemia can not only result in deterioration of parathyroid function but also affect bone mineral content [25].

Our results suggest that women with long-standing but otherwise uncomplicated Type I diabetes could be at increased risk of developing osteoporosis especially at the hip. There is evidence of increased bone resorption in the group of women with Type II diabetes. This study also shows abnormalities in the calcium/magnesium/vitamin D axis which could further compromise bone metabolism in this group. Therefore additional prospective studies of measurement of bone mineral density in conjunction with assessment of bone turnover, calcium homeostasis and COL1A1 genotyping are recommended in diabetic women in order to further investigate the usefulness of these variables in the assessment of risk factors for osteoporosis and fractures in women with diabetes.

Acknowledgements. This work was funded by a grant from the National Osteoporosis Society (United Kingdom ) awarded to Dr G. Hampson.

\section{References}

1. Selby PL (1988) Osteopenia and Diabetes. Diabetic Medicine 5: 423-428

2. Thomas DM, Ng KW, Best JD (1997) Insulin and bone: a Clinical Scientific Review. Endocrinol Metab 4: 5-17
3. Hui SL, Epstein S, Johnston CC (1985) A prospective study of bone mass in patients with Type I diabetes. J Clin Endocrinol Metab 60: 74-80

4. Rico H, Hernandez ER, Cabranes JA, Comez-Castresana F (1989) Suggestion of a deficient osteoblast function in diabetes mellitus: the possible source of osteopenia in diabetics. Calcif Tissue Int 45: 71-73

5. McNair P, Christiansen C, Christensen MS et al. (1981) Development of bone mineral loss in insulin-treated diabetes: a $1 \frac{1}{2}$ years follow-up study in sixty patients. Eur J Clin Invest 11: 55-59

6. Verhaeghe J, Suiker AMH, Visser WJ, Van Herck E, Van Bree R, Bouillon R (1992) The effects of systemic insulin, insulin-like growth factor-I and growth hormone on bone growth and turnover in spontaneously diabetic BB rats. J Endocrinol 134: 485-492

7. Bertelloni S (1992) The parathyroid hormone - 1, 25 - dihydroxyvitamin D endocrine system and magnesium status in insulin-dependent diabetes mellitus: current concepts. Magnesium Research 5(1):45-51

8. Schedl HP, Heath H, Wenger J (1977) Serum calcitonin and parathyroid hormone in experimental diabetes: effects of insulin treatment. Endocrinology 103: 1368-1373

9. Nyomba BL, Verhaeghe J, Thomasset M, Lissens W, Bouillon R (1989) Bone mineral homeostasis in spontaneously diabetic BB rats: abnormal vitamin $\mathrm{D}$ metabolism and impaired active intestinal calcium absorption. Endocrinology 124: 565-572

10. Frazer TE, White NH, Hough S et al. (1981) Alterations in circulating vitamin $\mathrm{D}$ metabolites in the young insulin-dependent diabetic J Clin Endocrinol Metab 53: 1154-1159

11. Pocock NA, Eisman JA, Hopper JL, Yeates MG, Sambrook PN, Ebert S (1987) Genetic determinants of bone mass in adults: a twin study. J Clin Invest 80: 706-710

12. Morrison NA, Qi JG, Tokita A et al. (1994) Prediction of bone density from vitamin D receptor alleles. Nature 367: 284-287

13. Pocock M (1995) Vitamin D receptor alleles and osteoporosis : a contrasting view. J Bone Miner Res 10: 1294-1297

14. Grant SFA, Reid DM, Blake G, Herd R, Fogelman I, Ralston SH (1996) Reduced bone density and osteoporosis associated with a polymorphic Sp1binding site in the collagen type $1 \alpha$ I gene. Nature Genetics 14: 203-205

15. Van D, Daele PLA, Stolk RP et al. (1995) Bone density in non-insulin dependent diabetes mellitus. Ann Intern Med 112: 409-414

16. Shore RM, Chesney RW, Mazess RB, Rose PG, Bargman GJ (1981) Osteopenia in juvenile diabetes. Calcif Tissue Int 33: 455-457

17. Kayath MJ, Dib SA, Vieira JGH (1994) Prevalence and magnitude of osteopenia associated with insulin-dependent diabetes mellitus. J Diabetes Complications 8: 97-104

18. McNair P, Madsbad S, Christiansen C, Faber OK, Transbol I, Binder C (1978) Osteopenia in insulin treated diabetes mellitus. Its relation to age of onset, sex and duration of disease. Diabetologia 15: 87-90

19. Wynn A and Wynn M (1995) Body mass index and nutrition in the aetiology of osteoporosis. J Nutr Environ Med 5: 41-53

20. Silberberg R (1986) The skeleton in diabetes mellitus: a review of the literature. Diabetes Res 3: 329-338

21. Verhaeghe J, Van Herck E, Visser WJ et al. (1990) Bone and mineral metabolism in BB rats with long-term diabetes. Diabetes 39: $477-482$

22. Gregorio F, Cristallini S, Santeusanio F, Filipponi P, Fumelli P (1994) Osteopenia associated with non-insulin-dependent diabetes mellitus: what are the causes? Diab Res Clin Pract 23: 43-54

23. Levin ME, Boisseau VC, Avioli LV (1976) Effects of diabetes mellitus on bone mass in juvenile and adult-onset diabetes. $\mathrm{N}$ Engl J Med 294: 241-245

24. McNair P, Christensen MS, Madsbad S, Christiansen C, Transbol I (1981) Hypoparathyroidism in diabetes mellitus. Acta Endocrinol 96: 81-86

25. Saggese G, Fedrico G, Bertelloni S, Baroncelli GI, Calisti L (1991) Hypomagnaesemia and the parathyroid hormone - vitamin D endocrine system in children with insulin - dependent diabetes mellitus: effects of magnesium administration. J Paediatr 118: $220-225$ 